\title{
Autobiographisches von Jehan Molinet.
}

Was wir von J. Molinets Leben wissen, ist so wenig, dals der Abdruck des nachfolgenden Gedichtes mit dem unumgänglichen Kommentar sich von selbst rechtfertigt. ${ }^{1}$ Den Text entnehme ich den Faictz et dictz de feu de bonne memoire maistre Jehan Molinet, Paris 1531, fol. XLVI-XLIX. Die Abschrift besorgte mir Herr Dr. J. Horváth.

Complainte pour le trespas de Madame Marie de Bourgoigne.

I.

En temps que pert Aurora ses grans pleurs,

Que Boreas mect jus ses grans souffletz,

Que Zephyrus nourrist plantes et fleurs,

Et que Flora prepare ses couleurs

Pour revestir arbresseaulx genteletz,

Où vont chantant les petits oiseletz:

Je fuz bany de toute esjouyssance.

Chascun n'a pas le temps à sa plaisance.

2.

En ses palus Hecate me rua;

Stix en ses flotz de pied coy m'atendit;

En larmes d'oeil que Concitus sua

Je fuz plongé, Acheron m'argua,

Et Flegeton en son feu m'estendit.

Mais Belsamen (sic) qui mon cas entendit,

Me respita du danger qui me nuit.

N'est si long jour que ne vienne à la nuyct.

1 Die älteren biographischen Studien und einzelnen Beiträge zu Molinets Leben sind so gut wie unzugänglich geworden. Vgl. U. Chevalier, Répertoire. Die Brochüre von Alph. Lefebvre, Vie et commune origine de Jehan Molinet, le Bolognois, et de Jehan Le Maire, le Belgeois, Boulogne s. M. I90I, bringt neben vielem Veralteten und Falschen als Neues nur den Nachweis einer Familie Le Maire du Moulinet in baillage von Desvres (1519 Jean Le Maire sieur du grand Moulinet, 1557 Jehan le Maire dit du Moulinet, 1572 Philippe Le Maire écuyer sieur du Moulinet). Die Zugehörigkeit der beiden Dichter zu dieser Familie ist durchaus zweifelhaft; denn der Nachweis bedeutet nur soviel, dafs im 16. Jahrh. die Familie 'Meyer' irgendwo die 'grofse Mühle' als Lehen verwaltete.

Zeitschr. E. rom. Phil. XXVI. 


\section{3.}

Monté comme ung petit conte ou vidame, Mieulx que celluy qui desire fort une, Je me rendy, desirant salut d'ame, Vray pelerin à l'humble vierge dame Qui renommee est de bonne fortune. Mais quant ce mal qui tant me defortune Vueil appaiser, il s'acroist plus d'ung tiers. Maulvaise herbe croist tousjours voulentiers.

4.

Je m'aprochay d'une tres grosse ville Pour mon devot, sainct voyage entamer; Tous biens y sont conduictz par loy civille, Son nom est fort bruyant, gent et non ville; Pour le present Bruges se fait nommer. Tout $y$ arrive et par terre et par mer. C'est du païs la resplandissant lume.

Les beaux oyseaulx congnoist on à la plume.

5.

Pour recepvoir pellerins telz ou quelz

Et vivre en paix trop mieulx que en ville franche, Il $y$ avoit plusieurs grans beaux hostelz Sortis de lis, de chappelle et d'autelz Et de liesse, en lieu d'avoir souffrance. Loger cuiday au noble escu de France; Mais l'hoste estoit rioteux et sauvage. Envis chet on de franchise en servage.

6.

Aux trois luppars y avoit grant envoy; L'hoste estoit riche d'avoir et de bagage. Je heurte a l'huis. Autre part je m'en voy Querir logis; car ame je n'y voy Qui vueille ou sache entendre à mon langage. Il y a port de mer et bon pesquage Pour riches gens qui vivent a cher coust. Mais povres gens n'ont partout point d'escout.

7.

Beant en hault, veiz l'escu de Bretaigne, Maison fort haulte, au siecle renommee.

Mais moy, qui n'ay vaillant une chastaigne, N'y puis avoir (sans monter la montaigne) Logis ouvert ne chandelle allumee. Toutes fois quant j'en perceuz la fumee. J'en euz soulas et joyeuse attendue. Chose qui plaist est dे demy vendue. 
8.

Je fuz jadis, comme cil qui s'avoye

De pourchasser l'hostel de grant demaine, Tresbien logé à l'escu de Savoye;

Mais le noble hoste s'en est allé sa voye.

Mort y a pris hoste et hostesse humaine;

Je ne scay plus quel estat on y maine.

Les enfans sont dispers et eslongez.

Par guerre sont toutes gens mal logez.

9.

Pour moy loger, vins à l'escu d'A rtois.

Mais le logis estoit plain de gens d'armes, Et le propre hoste, humble, sage et courtois, Ne possedoit que une chambre et ung toictz $Q$ u'il deffendoit encore à force d'armes.

Et quant je veiz tant d'armes, de guisarmes, Je me garday de bien passer par là.

Car trop envis meurt qui apris ne l'a.

10.

De loing perceuz l'ostel de Ravestain, Cler et luysant comme estoille ou beau cierge; Le bruyt en est partout assez haultain. Mais j'entendis par quelque secretain Que l'hoste avoit pour ceste nuyct sa charge: Car il estoit gardien et concierge

De deux joyaulx de precieuse espreuve.

Qui bien se garde on dit que bien se treuve.

11.

Je reiz l'hostel de Sainct.Pol, et l'ymage

Doré d'honneur et de nobilité,

De hault estocq et de puissant lignage.

Mais l'hoste estoit (dont ce fu grant dommage)

Maladieux et fort debilité.

Neantmoins il fut assez babilité

De moy loger, se mieux je ne receuvre.

Le bon vouloir est reputé pour œuvre.

12.

A la dolloyre honorable et entiere Trouvay l'hostel et bruyt hault eslevé. L'ung des hostes gardoit nostre frontiere, Et l'autre estoit pour certaine matiere Au noble hostel de Bretaigne arrivé.

Et quant je n'ay quelque ung des deux trouvé, Je m'en partis sans illecq arrester.

Hastif besoing fait la vieille trotter. 
13.

Au Toison d'or y estoit grant repaire. Tresredoubtez princes resplendissans, Roys s'y logent, l'empereur y repaire, Ducz et barons choisis, plus d'une paire, Accompaignez de chevaliers puissans. Fort diligens sont leurs obeissans De les servir et de genoulx ployer. Qui sert bon maistre, il attend bon loyer.

14.

De chevaliers estoit le logis plain. Je reconguenz les armes et les cris: Anthoing, Lannoy, La Grutuze, Lalain, Romont, Chimay, Nasso, Bevres, Walain, Ligne et Bousut, nouvellement escriptz. Aultres y sont de hault loz et de pris; Mais je ne puis regarder à tous boutz. Impossible est de bien complaire à tous.

15.

Au noir lyon sounoient les musettes, On y trouvoit vivres, biere et harens, Et à crogner de bien dures noisettes, Qui deviendront framboises et rosettes Se beau soleil se troque sur les rens. Et se Walons sont unys aux Flamens, Par bonne amour, ame ne les peult nuyre. A peu de vent fait on son fuseau ruyre.

16.

Au chappeau rouge ung bong logis trouvay Plain de legatz et de gens fort lettrez.

Tant en $y$ eust que bref m'en retournay. Là fust logé l'evecque de Tournay; Tres reverendz peres y sont entrez.

Aux bons docteurs, aux evesques mittrez Ja ne sera la maison refusee.

Commencement n'est point si tost fusee.

17.

Du chappeau rouge arrivay à la crosse, Où les manans ne parlent que latin.

Henri de Bergues, ung prelat sans reproche, Qui de Cambray le chappeau rouge approche, $Y$ fut loger par ung lundy matin.

Nous y verrons l'abbé de Saint-Bertin Ung jour loger à son plus hault dongeon. Maille a maille faict on le haubergeon. 
18.

Je me tiray vers le perron du Liege, Querant logis où gueres ne me tins:

Car l'hoste estoit, ensemble le colliege, En grant danger de perdre hostel et siege Par le discord d'aucuns maulvais mutins: Quant je perceuz eslever les hutins: Je picque arriere, en musant d'ung cousin. Au monde n'est tencer que de voisin.

19.

Parlant du bar et du grant elephant, Il me souvint de l'escu de Bourgoigne, Lequel Philippes et Charles son enfant Tindrent en haut estat, tant triumphant Que bruyt en fut jusques en Castelloine. Et maintenant par la guerre qui hongue, Le cerf vollant y plante son escu. A l'enfourner fait on le pain cocu.

20.

De pain, de vin, de fourmage et de table Estoit sorty quand la guerre $y$ survint; On y trouvoit court ouverte et notable Cousteau a table et large en ronde table A tous passans il y a des ans vingt. Mais puis le jour que nouvel hoste $y$ vint, Nous n'y peumes ne loger ne repaistre. J.e dernier veult tousjours estre le maistre.

2 I.

Finablement emprès l'aigle romain Trouvay logis nommé l'hostel d'Austriche, Nouvel essours par forte et puissant main. L'hoste estoit noble et courtois et humain, Sage, prudent et moiennement riche. L'hostesse aussi, de vertu la nourrice, Estoit puissante et terrienne grande.

On doit donner à tel saint tel offrande.

22.

Pour le grant bien que dire j'en ouy, Je demanday logis et retenance:

Esperant d'estre à demy resjouy.

Mais de l'hostel je n'ay gueres jouy, Quant triste fuz et plain de grant tennance; J'aperceuz gens de simple contenance, Chargez de pleurs et de dueil attournez. En peu de jours sont les vens retournez. 
23.

Car la tres bonne et gracieuse hostesse, Riche de meurs, gente et bien composee Noble de sang, naissant de la haultesse De roys, de ducz, de conte et de contesse, Estoit ce jour du siecle trespassee.

Lors fut la fleur de noblesse cassee, Des Bourguignons l'appuy et le baston. Plus vault tresor, et plus tost le pert on.

24.

Ce jour versa sur la tres redoubtee Maison d'Austrice ung douloureux tempeste. Quoy qu'elle soit haute et bien charpentee, Son bourg fondit, sa court en fut gastee, Son bruyt cessa, elle perdit sa feste, Et sa chapelle; il est tout manifeste Que le propre hoste en fut tout eperdu. Quant le chef fault, tout le corps est perdu.

25.

Son corps gisoit sur couche bien paree, Environné de maint grant personnage Plourant sa mort à nulle accomparee, Et qui jamais ne sera reparee Par hault crier, par chant, ne par tonnage. Elle nous laisse en la fleur de son aage. Trop tard, helas! on le voit par espreuve:

On scait qu'on pert, mais on ne scait qu'on treuve.

26.

Entre les haultz hostelains d'excellence,

Faisant grant dueil pour l'ennuy qui nous blesse,

Estoit honneur, grace, benivolence,

Misericorde, amitié, sens, prudence,

Fidelité, magnificence, humblesse,

Devotion, courtoisie, noblesse,

A vec pitié qui plouroit larmes d'oeil.

Chascune ville à son tour plaint son dueil.

27.

Pendant le temps que l'ung tordoit ses mains, Battant son pis par ung tres grant courroux, Et que l'autre qui n'en pensoit pas mains, Jectans regretz piteux et inhumains, Avoit ses doigtz et ses cheveux desroulx:

Dame Noblesse aux blonds cheveux, non roux, Fort soupirant, voulut ses motz tyssir.

De cueur dolent ne peut grant joye yssir.

(Suit la plainte de Noblesse.) 
Den Anlafs zu der vorstehend abgedruckten Dichtung gab Molinet der Tod Marias von Burgund, welcher am 25. März 1482 in Brügge erfolgte. Auf dieses Ereignis zielen die Eingangsworte: Au temps ... que Flora prepare ses couleurs: je fuz banny de toute esjouissance. Trost sucht der Dichter in der Erinnerung an seine früheren Prüfungen (Str. 2). Er stellt sich vor als einen Pilger, der Glück suchend zur heiligen Jungfrau wallfahrtet, dem aber jeder Versuch sich dem Mifsgeschick zu entwinden in dreifaches umschlägt (Str. 3). Seine Fahrt beginnt er mit Brügge (pour mon devot, saint voyage entamer, Str. 4) offenbar im Hinblick auf das hier erfolgte Ableben der Herzogin. In dieser reichen Stadt sieht er eine Reihe Gasthäuser, mit aller Bequemlichkeit ausgestattet, und überlegt, in welchem er Unterkunft suchen soll. Dies ist die Filtion, die sich durch das ganze Gedicht zieht und auch im einzelnen den Ausdruck bestimmt.

Des Verfassers erster Gedanke richtet sich nach Frankreich: Loger cuiday au noble escu de France; mais l'hoste (Ludwig XI.) estoit rioleu.x et sauvage (Str. 5). In England (aux trois luppars) herrscht Reichtum; aber französisch versteht man dort nicht: mais ame je n'y voi qui vueille ou sache entendre a mon langage (Str. 6). Oben auf der Höhe steht das 'bretagnische Wappen'; man mülste den Berg erklimmen; Molinet wagt es nicht, freut sich aber schon am aufsteigenden Rauch (Str. 7). - „Ich war vormals, - und hier finden wir die erste positive Angabe, - gut untergebracht im 'savoyischen Wappen', aber der Tod hat Wirt und Wirtin abberufen, die Kindern sich zerstreut und fern" (Str. 8). Die Anspielung kann sich nur auf Amatus IX. von Savoyen beziehen; dieser starb 1472 am Tag vor Ostern; seine Gemahlin, Yolande von Frankreich, folgte ihm 1478 nach; als ihr Bruder, malste sich Ludwig XI. die Vormundschaft über ihre Kinder zu, liefs die beiden jüngsten in Frankreich erziehen, und hatte eben auch den ältesten nach Lyon bringen lassen, wo derselbe am 24. April 1482 einer Erkältung erlag. Dic Angaben dieser Strophe lassen an Klarheit nichts zu wünschen übrig; wir erfahren auf das bestimmteste, dafs Molinet vor Ostern 1472 am savoyischen Hof in Dienst gewesen ist. Dies ist als das erste gesicherte Datum festzuhalten.

Der Tod des Herzogs von Savoyen läfst also den Dichter von neuem ohne Obdach. Wohin gehen? Artois ist von Truppen erfüllt, dafs dem Hausherrn selbst nur eine Dachkammer bleibt (Str. 9). In der That hatte Karl der Kühne den Tod Guyennes (12. Mai 1472) zum Vorwand genommen, um seinem Bruder Ludwig XI., dem man den Tod schuld gab, den Krieg zu erklären; während er selber Beauvais belagerte und die Normandie plündernd durchstreifte, verwüstete der Connetabel von Frankreich Picardie und Artois. ${ }^{1}$ - Berühmt war das Haus Ravenstein, aber durch

1 Artois gehörte dem Burgunder. Am 25. Juli 1472 starb der letzte Artois-sans-terre, Karl Graf von Eu und Saint-Valery; es ist nicht anzunehmen, dafs Molinet an ihn dachte. 
einen Schreiber hört Molinet, dafs der Hausherr eben sein Amt versehen mufs als Wächter zweier kostbarer Kleinodien (Str. 10). Gemeint ist Adolph von Cleve, Herr von Ravenstein; der Sinn der Anspielung bleibt aber dunkel, es sei denn dals sie auf spätere Jahre geht, wo der Herr von Ravenstein die Pflege Philipps des Schönen (geb. I478) und Margaretas (geb. 1480) hatte. - Ein vornehmes Haus ist auch Saint-Pol, und der Wirt, obgleich krank und schwächlich, war gern bereit unseren Dichter aufzunehmen, falls er nicht besser fände (Str. I I). Die Worte zielen auf Ludwig von Luxemburg, Grafen von Saint-Pol und Connetabel von Frankreich, dessen Stern im Sinken begriffen war. Lange hatte er seine Stellung zwischen Frankreich und Burgund zu einem gewagten Intrigenspiel ausgebeutet; sein Geschick besiegelte er, als er 1473 Saint-Quentin durch einen Handstreich wegnahm; Jahrs darauf erklärten ihn der König und der Herzog als ihren gemeinsamen Feind, und es verschlug nichts, dafs Ludwig XI. ihn gleich darauf zu Verhandlungen mit Karl dem Kühnen verwendete; 1475 ward er von Karl festgenommen, nach Frankreich ausgeliefert und am 19. Dezember in Paris enthauptet. - Im 'Beil' (a la dolloyre) findet der Dichter beide Wirte abwesend, der eine hütete die Grenze, der andere war zum 'bretagnischen Hof' gegangen in bestimmten Geschäften (Str. I2). Drei rote Beile auf Silberfeld führten die Herren von Croy als Erben von Renty. Im letzten Lebensjahr Philipps des Guten hatten sich diese mit dem Erbprinzen überworfen, und als dieser die Herrschaft antrat, gingen sie nach Frankreich über; noch 1475 im Friedensabschlufs mit Frankreich liefs Karl den älteren Zweig ausdrücklich ausnehmen; mit dem jüngeren war die Versöhnung schon früher erfolgt, Karl erhob Philipp zum Grafen von Chimay, und dieser diente ihm sowohl im Heer als auf Gesandtschaften, er begleitete ihn auf dem Feldzug gegen die Schweiz und Lothringen, und Anfang 1481 war er in der Bretagne als Gesandter; sein Bruder war Michel von Sempy. Diese beiden dürften hier gemeint sein; genauer läfst sich die Anspielung nicht ausdeuten, weil der Zeitpunkt wenig bestimmt ist. - Aus diesen Strophen (9-12) ergiebt sich also soviel, dals Molinet, nachdem er den savoyischen Dienst verlassen, sich vergeblich bemüht hat in Artois, beim Herrn von Ravenstein, beim Connetabel von SaintPol und bei den Herren von Croy (oder eher Chimay) Anstellung zu finden. Am ehesten hätte ihn der Connetabel aufgenommen; und dieser mehr oder minder glückliche Versuch mufs zwischen 1472 und 1475 stattgefunden haben.

Aber folgen wir der Dichtung weiter: Grolser Zudrang war beim 'goldenen Vliefs; Könige, der Kaiser, Herzöge, Barone sind dort untergebracht und werden fleifsig bedient. Von Rittern war die Wohnung voll; der Verfasser erkennt ihre Wappen und Feldrufe: Anthoing, Lannoi, etc. (Str. I3. 14). - Die Versorgung, die er suchte, fand also Molinet beim Orden des goldenen Vliefses. Die Ritter, die er erwähnt, lebten alle noch, als die Dichtung 
schrieb: Jean de Melun, Herr von Anthoing, der Senior des Ordens; Jean de Lannoi, Herr von Molembais; Louis de Bruges, Herr von La Grutuse (Gruythuyse); Josse de Lalain, Herr von Montigny; Philipp von Savoyen, Graf von Romont, der im Krieg zwischen Burgund und der Schweiz eine grolse Rolle gespielt und schliefslich auf seine Apanage im Wadtland hatte verzichten müssen; dann Philipp von Croy, Graf von Chimay; Engilbert, 'Graf von Nassau; Philipp von Burgund, Herr von Beveren, der Verteidiger Saint-Omers; Jean de Berghe, Herr von Walhain; Jean de Ligne, Herr von Barbançon, der die Erbin von Aremberg heiratete; endlich Jean de Hennin, Herr von Bossut. Die letzteren nennt Molinet mit Recht nouvellement escriptz; denn sie waren erst beim Ordensfest von $148 \mathrm{I}$ in die Zahl der Ritter aufgenommen worden. Der 'schwarze Löwe', den die folgende (15.) Strophe erwähnt, ist das Wappen von Flandern, Hennegau und Namur; dunkel ist der Sinn der Tanzmusik, der harten Haselnüsse, die Himbeeren und Rosen werden sollten, wenn die Sonne auf den Plan tritt. Dunkel ist auch die Beziehung dieses Wappentiers zum goldenen Vliefs; denn auf letzteres beziehen sich wieder die folgenden Strophen: Der 'rote Hut', wo der Bischof von Tournay logiert (Str. I6), bezeichnet Ferri von Clugny, seit 1474 Bischof von Tournay und wie sein Vorgänger Kanzler des Ordens vom Vliefs; am I5. Mai 1480 wurde er Kardinal vom Titel von San Vitale. Sein Nachfolger im Kanzleramt ward Heinrich von Berghen, der am 17. Mai I 480 (par un lundi matin) Bischof von Cambray wurde, un prélat sans reproche, wie auch die Gallia christiana bezeugt: 'litterarum scientia, vitae munditia, honestate morum, multiplicibus virtutum donis ornatum'; er war der bekannte Gönner Erasmus', und es läfst sich denken, dafs man in seinem Hause vornehmlich Latein hörte, und ein sehr gewähltes. Als Kanzler sollte ihm später Jean de Lannoy, Abt von Saint-Bertin, ersetzen (vgl. Str. 17 Schluss).

Fassen wir zusammen, was diese Strophen (13-17) andeuten, so ergiebt sich, dals Molinet beim Orden des goldenen Vlielses angestellt gewesen sein mufs; in welcher Eigenschaft ist schwer zu sagen. Als Sekretär des Kanzlers vielleicht; möglicherweise als Wappenherold, und zwar nicht als Toison d'Or, denn das Amt des Wappenkönigs versah noch Lefívre, aber sehr wohl als llerold von Flandern oder eher Hennegau (noyr lyon). Welcher Natur auch sein Amt war, vermuten lärst sich, dafs er dasselbe als Nachfolger George Chastellains ( $\dagger$ 20. März I 475) bekleidete; auch dieser scheint zum Orden in einem gewissen, bisher nicht näher bestimmten Verhältnis gestanden zu haben.

Was Molinet veranlaiste diese Stellung aufzugeben und sich in Lüttich Unterkunft zu suchen (Str. I8), ist nicht zu ersehen. Hier fand er lauter Aufruhr und Unsicherheit; Ludwig von Bourbon, der Bischof, hatte sich mit der Stadt nie vertragen, und der Zwist hatte beiden Teilen schwer geschadet. Wie gering sein Anhang war, zeigte sich, als Wilhelm von der Marck, der 'Eber der Ar- 
dennen', im Jahre 1482 gegen ihn zog und ihn am 30. August elend erschlagen liefs. Hier war also in der That nicht gut wohnen.

Wohin nun jetzt? Zur 'Barbe' oder zum 'grolsen Elephanten'? Die Barbe (bar) führten die Grafen von Bar aus dem Hause Mömpelgard; Anspruch auf die Grafschaft hatte damals der gute König René von Anjou; in der Hand hatte sie sein Enkel René Herzog von Lothringen, und dieser schmüclte als Vaudémont seinen Helm mit zwei Büffelhörnern, die man nach der heraldischen Auffassung des ausgehenden 15. Jahrhunderts wohl auch als Elephantenrüssel (proboscides) auffassen konnte; denn die Elephantenrüssel scheinen ja nur eine Mode-Auslegung der stilisierten Hörner zu sein. Jetzt endlich erinnert sich der Dichter des 'burgundischen Hofs', der unter Philipp und Karl in so hohem Ruhme stand, wo aber jetzt, bei den herrschenden Kriegszeiten, der 'fliegende Hirsch' sein Schild aufpflanzt. Ja vor etwa zwanzig Jahren war hier aller Ueberflufs zu finden und gastliche Aufnahme, bis der neue Wirt kam: mais puis le jour que nouvel hoste y vint, nous n'y peumes ne loger ne repaistre (Str. 19. 20). Der 'fliegende Hirsch' ist eine heraldische Bezeichnung für die Franzosen, so gebraucht es J. Lemaire in den Chansons de Namur (Str. I3), synonym mit porczespicz, dem Wappen Ludwigs XII. als Orléans. Nach Stecher (Euvres de J. Lemaire IV, 358) bezeichnet es Bourbon, speziell den Connetabel Karl von Bourbon-Montpensier. Belege dafür kenne ich nicht; andererseits habe ich auch das eigentliche Wappen der Valois, an die man denken könnte, nicht ausfindig machen können. Es zeigt sich also, dafs Molinet unter Karl dem Kühnen keine Verwendung im herzoglichen Dienst fand; es ist auch begreiflich, da Karl seit 1475 Niederburgund nicht wiedersah, sondern verblendet seinem Verhängnis folgte. Erst zuletzt beim 'Straufsenwirt' in der Nähe des 'römischen Adlers' findet Molinet Unterkunft (Str. 21), d. h. bei Maximilian von Oesterreich (das Wortspiel mit austruche ist nicht neu), dem Sohn des Kaisers, und bei seiner Gemahlin Maria von Burgund. Kurz war aber die Freude, denn die edle Frau starb, wie gesagt, am 25. März 1482.

Fassen wir das Ergebnis zusammen!

Jehan Molinet war 1435 in Desvres bei Boulogne s. M. geboren. Den Geburtsort erwähnt die lateinische Grabschrift: Me Molinet peperit Divernia Boloniensis; die Jahreszahl ergiebt sich daraus, dals Molinet 1507 im Alter von 72 Jahren starb (s. Lemaire, (Euvres ed. Stecher IV, 52I). Seine Bildung erhielt der spätere Historiograph in Paris; Parisius docuit, sagt das Epitaphium, und Lemaire nennt seinen Verwandten stets maistre Jehan Molinet. Wie es scheint, blieb er dann einige Zeit im Kollegium des Kardinal Lemoine als Amtsschreiber, denn so dürfte wohl das escrivain du cardinal Le Moine zu verstehen sein, 'd'après une lettre de Molinet aux logiciens du collège de Montaigu à Paris, résumée par Chifflet' (vgl. E. Roy, les lettres de Noblesse du poète Jehan Molinet in 
Revue de phil. fr. et prov. p. p. Clédat IX, I9-2I. I 895). Später trat er in den Dienst des Herzogs Amatus IX. von Savoyen und verblieb hier bis zu dessen Tod, Ostern 1472. Nach dem Norden zurückgekehrt, findet er den Krieg zwischen Frankreich und Burgund entbrannt; gern hätte ihn der Connetabel von Saint-Pol aufgenommen, Molinet fand aber besser. Er trat beim Orden des goldenen Vliefses in Dienst, sei es als Sekretär des Kanzlers, sei es eher als Herold von Flandern oder Hennegau, vielleicht als Nachfolger Chastellains (1475); diese Funktion behielt er möglicherweise bis 1481 oder auch länger, denn sie liefs sich mit anderen kumulieren. Erst unter Maximilian trat Molinet direkt in den herzoglichen Dienst, und zwar - wie sich vermuten läfst in dem seit Chastellains Tod erledigten Amt des Hofgeschichtsschreibers. In diesem Amt verblieb er bis zu seinem Ende: „en ce mois d'aoust, sagt Lemaire an der angeführten Stelle zum Jahr I 507 (Euvres IV, 52I), par ung lundy XXIII d'icelui, en la bonne ville de Vallenciennes termina sa vie par mort aggravé de vieillesse et de maladie en l'aaige de LXXII ans, le chief et souverain de tous les orateurs et rhetoriciens de nostre langue gallicane, c'est assavoir maistre Jehan Molinet, chanoine de la Salle le conte, renommé par tous les quartiers d'Europe ou ladicte langue a lieu: lequel dans son jeune aaige adonné au service de musicque et de rhetoricque, fut premierement disciple et depuis successeur de messire George Chastellain, indiciaire et historiographe de la maison de Bourgoigne, et continua les cronicques d'icelle depuis le siege tenu devant Nuz (d. i. Neu[s, 1474) par le grand duc Charles jusques au merveilleux nauffrage et abordement en Engleterre du feu roy Philippes tirant en Espaigne (1505)." Das hier erwähnte Kanonikat dürfte Molinet erst als Witwer erhalten haben; denn er war verheiratet und hinterliefs zwei Söhne, Augustin, Kanonikus von Condé, und Balthazar, Historiograph Karls V. Am 1. April 1503 war er auch vom Kaiser geadelt worden. Sein unmittelbarer Nachfolger im Amt wurde bekanntlich Jehan Lemaire, für dessen Laufbahn das Leben seines Verwandten in mancher Hinsicht vorbildlich genannt werden kann.

Pi1. Aug. Becker. 